\title{
Structure, mass and distance of the Virgo cluster from a Tolman-Bondi model
}

\author{
P. Fouqué ${ }^{1,2}$, J. M. Solanes ${ }^{3}$, T. Sanchis ${ }^{4}$, and C. Balkowski ${ }^{5}$ \\ 1 Observatoire de Paris-Meudon DESPA, 92195 Meudon Cedex, France \\ 2 European Southern Observatory, Casilla 19001, Santiago 19, Chile \\ 3 Departament d'Enginyeria Informàtica i Matemàtiques, Escola Tècnica Superior d'Enginyeria, \\ Universitat Rovira i Virgili, Carretera de Salou, s/n; 43006 Tarragona, Spain \\ e-mail: jsolanes@etse.urv.es \\ 4 Departament d'Astronomia i Meteorologia, Facultat de Físiques, Universitat de Barcelona C/Martí i Franqués 1, \\ 08028 Barcelona, Spain \\ e-mail: tsanchis@am.ub.es \\ 5 Observatoire de Paris-Meudon DAEC, 92195 Meudon Cedex, France \\ e-mail: Chantal.Balkowski@obspm.fr
}

Received 3 April 2001 / Accepted 31 May 2001

\begin{abstract}
We have applied a relativistic Tolman-Bondi model of the Virgo cluster to a sample of 183 galaxies with measured distances within a radius of 8 degrees from M 87. We find that the sample is significantly contaminated by background galaxies which lead to too large a cluster mean distance if not excluded. The Tolman-Bondi model predictions, together with the HI deficiency of spiral galaxies, allows one to identify these background galaxies. One such galaxy is clearly identified among the 6 calibrating galaxies with Cepheid distances. As the TolmanBondi model predicts the expected distance ratio to the Virgo distance, this galaxy can still be used to estimate the Virgo distance, and the average value over the 6 galaxies is $15.4 \pm 0.5 \mathrm{Mpc}$. Well-known background groups of galaxies are clearly recovered, together with filaments of galaxies which link these groups to the main cluster, and are falling into it. No foreground galaxy is clearly detected in our sample. Applying the $B$-band Tully-Fisher method to a sample of 51 true members of the Virgo cluster according to our classification gives a cluster distance of $18.0 \pm 1.2 \mathrm{Mpc}$, larger than the mean Cepheid distance. Finally, the same model is used to estimate the Virgo cluster mass, which is $M=1.2 \times 10^{15} M_{\odot}$ within 8 degrees from the cluster center $(2.2 \mathrm{Mpc}$ radius), and amounts to 1.7 virial mass.
\end{abstract}

Key words. galaxies: clusters: individual: Virgo - galaxies: distances and redshifts - galaxies: elliptical and lenticular - galaxies: fundamental parameters - galaxies: spiral - cosmology: distance scale

\section{Introduction}

In 1990, Fouqué et al. (1990) derived an unbiased distance to the Virgo cluster, based on a complete sample of 178 spiral galaxies, using the $B$-band Tully-Fisher relation (Tully \& Fisher 1977). A previous similar study based on 110 spiral galaxies ( $\mathrm{Sa}-\mathrm{Sm}$ ) was published by KraanKorteweg et al. (1988). Soon after, Teerikorpi et al. (1992, hereafter T92) suggested that this distance determination may have been contaminated by the inclusion of background galaxies into the complete sample, although we a priori excluded galaxies generally attributed to the background $\mathrm{M}$ group and $\mathrm{W}$ cloud.

The study of the structure of the Virgo cluster starts with de Vaucouleurs (1961), who identifies the

Send offprint requests to: P. Fouqué, e-mail: pfouque@eso.org southern extension (Virgo cloud X), and the wing (Virgo cloud W). This study is extended in de Vaucouleurs \& de Vaucouleurs (1973) who separate the Virgo I cluster (Virgo E, S and S') from the Virgo II cloud complex (composed of Virgo V, X and Y) and the background W cloud (composed of Virgo Wa, Wb and W' sub-groups). Paturel (1979) applies taxonomy to disentangle these various components. Then, Tully (1982) discusses the separation between the Virgo cluster and its southern extension in the frame of the Local Supercluster. Later, Ftaclas et al. (1984) identify the M group and the N group. Pierce \& Tully (1988) use the Tully \& Shaya infall model (Tully \& Shaya 1984) to study the velocity-distance diagram of bright Virgo spiral galaxies. Binggeli et al. (1993) clarify the situation by listing galaxies from the VCC catalogue (1985, hereafter VCC) belonging to each of these clouds (W, W', M and southern extension). T92 list several 
regions in the velocity-distance diagram: A, B, C1, C2, and D. Yasuda et al. (1997) also study the 3-D structure of the cluster. Finally, Gavazzi et al. (1999, hereafter G99) use distance determinations to identify new groups, named $\mathrm{B}, \mathrm{E}, \mathrm{N}$, and $\mathrm{S}$.

Please note that a clarification of the nomenclature is highly desirable, as the same letters refer to totally different groups: A can mean the main Virgo cluster or refer to galaxies with high velocities in front of Virgo and falling into the cluster (T92); B can mean the concentration around M 49, or a background group in the same region (G99), or even a foreground expanding component (T92); E can refer to the elliptical component of the Virgo cluster or an eastern group (G99); N can be a group identified by Ftaclas et al. (1984) or a northern group in G99; $\mathrm{S}$ refers to the spiral component of the Virgo cluster or a southern group in G99; X can mean that the galaxy lies within the X-ray contours (Federspiel et al. 1998, hereafter F98) or belongs to the Virgo X cloud (de Vaucouleurs 1961)!

To investigate the structure of the Virgo cluster and determine the mean distance of the main cluster, we have used the Tolman-Bondi model of the cluster defined in Ekholm et al. (1999, hereafter E99) in the spirit of T92, for spiral galaxies whose distances were given by the Tully-Fisher relation in B-band by Ekholm et al. (2000, hereafter E00) or in $H$-band by G99, but adding the information given by the HI deficiency of Virgo spiral galaxies. We also used the same model for early-type galaxies whose distances were determined by the fundamental plane method in G99, or the recent Tonry's compilation of Surface Brightness Fluctuations distances (Tonry et al. 2001, hereafter T01).

Section 2 defines the observable parameters we will use for this study. Section 3 introduces the Tolman-Bondi model and lists its adopted parameters. Section 4 investigates how the discrepant Cepheid distance to NGC 4639 is well explained by our model, and computes the average Cepheid distance to Virgo. Section 5 applies our model to the determination of the Virgo cluster mass, and compares it to other determinations. Section 6 investigates the structure of the Virgo cluster and lists galaxies which do not belong to the main cluster according to our model. Section 7 compares the average distances to Virgo adopted in the various references we used to what our model predicts. Finally, Sect. 8 summarizes the main results of this paper.

\section{Observable parameters}

The selected sample has been extracted from the LEDA database. It consists of 584 galaxies within a radius of 8 degrees $(2.25 \mathrm{Mpc}$ at $16 \mathrm{Mpc})$ from M $87\left(\alpha_{2000}=12.51381\right.$, $\left.\delta_{2000}=12.3900\right)$ and known recession velocities smaller than $3000 \mathrm{~km} \mathrm{~s}^{-1}$. Unfortunately, not all these galaxies have distance estimates, and we therefore reduced the working sample to 125 late-type and 67 early-type galaxies, with distance measurements from G99, E00, F98 and

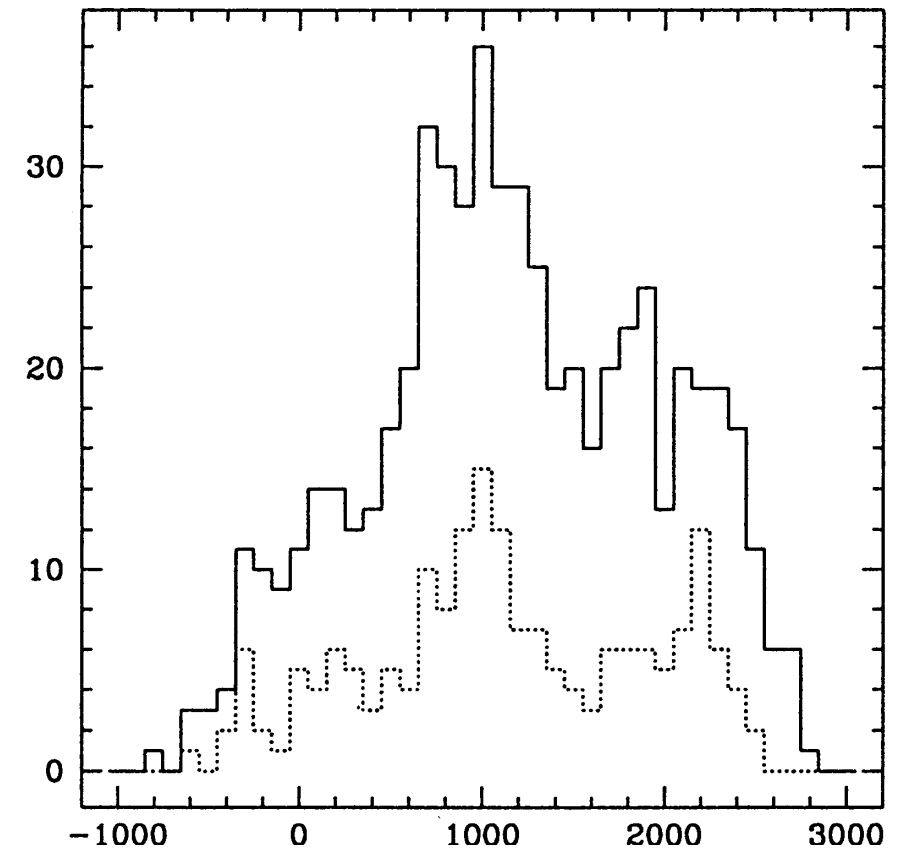

Fig. 1. Histogram of the recession velocities, in the Local Group reference frame, for the 584 galaxies within 8 degrees from M 87. The dotted histogram corresponds to galaxies with distance measurements.

T01. Let us describe in more detail some of the observable parameters collected for these galaxies.

\subsection{Coordinates and recession velocity}

These parameters are directly extracted from the LEDA database. Coordinates are given in the J2000.0 equinox, and helocientric velocities are corrected to the Local Group centroid using the Yahil et al. (1977) formula. A histogram of the corrected velocities for the full sample of 584 galaxies is presented in Fig. 1. It clearly shows more galaxies on the high-velocity side than expected from a gaussian distribution. A similar but smaller effect also seems apparent on the low-velocity side. For galaxies with distance measurements, the same features appear.

\subsection{Morphological types}

An accurate estimate of morphological types is important at least for two reasons: the HI deficiency parameter (see below) is calculated by comparing the HI content of a galaxy to the average HI content of an isolated galaxy of the same morphological type. Any error in the type therefore translates into a corresponding error in the HI deficiency estimate. Similarly, distances determined by E00 use the calibration of Theureau et al. (1997), where the intercept of the Tully-Fisher relation varies with the type. Any error in the type changes the galaxy distance value accordingly.

We used three sources of morphological type determinations: de Vaucouleurs et al. (1991, hereafter RC3), the VCC catalogue and van den Bergh et al. (1990). 
The quality of RC3 types depends upon its source, coded according to Table 3 of vol. 1 of the catalogue. If the morphological type has been measured on large reflector plates (code R), it is adopted; if it comes from PSS prints (codes $\mathrm{P}$ or $\mathrm{U}$ ), we adopt an eye average with the two other sources. The adopted morphological types differ therefore from those given in LEDA and used by E00.

We adopted a numeric morphological type according to the RC3 coding scheme. For distance measurements, we decided to restrict application of the fundamental plane and SBF methods to types between -5 and -1 ( $\mathrm{E}$ to L+) and of the Tully-Fisher method to types between 1 and 9 (Sa to $\mathrm{Sm}$ ). Therefore, 7 galaxies were excluded from the late-type sample (types -1, 0 and 10), and 2 galaxies were excluded from the early-type sample (NGC 4440 and NGC 4531, both of type 1). The final sample thus contains 118 spiral, 43 lenticular and 22 elliptical galaxies.

\subsection{Distances}

\subsubsection{Early-type galaxies}

For early-type galaxies, we have used the G99 compilation of distances, obtained from the fundamental plane method in $H$-band. According to these authors, their accuracy should be about $21 \%$. These distances are based on an assumed Virgo distance of $16 \mathrm{Mpc}$. To this compilation of 55 elliptical and lenticular galaxies, we added SBF distances from T01. It contains 35 early-type galaxies in the Virgo cluster area, of which 25 are common with G99. Tonry's distances are calibrated independently of any assumed Virgo distance, and the mean shift compared to G99 amounts to:

$$
\left\langle d_{\mathrm{T}}-d_{\mathrm{G} 99}\right\rangle=0.64 \pm 0.84
$$

after rejection of $\mathrm{NGC} 4638\left(d_{\mathrm{G} 99}=8.87 \mathrm{Mpc}\right.$ and $d_{\mathrm{T}}=$ $21.68 \mathrm{Mpc}$ ). As the mean difference of the two systems is not significant, we adopt an unweighted average of the two measurements as our distance measurement for this set of 25 early-type galaxies, and the uncorrected only available distance for the 40 other galaxies (30 from G99, 10 from Tonry). Please note that both references include more uncertain measurements: in G99, they are identified as being due to velocity dispersions smaller than $100 \mathrm{~km} \mathrm{~s}^{-1}$; in T01, they appear in a separate table, named "table.poor". We have kept these galaxies in our sample, but we generally exclude them from our statistical calculations.

\subsubsection{Late-type galaxies}

For late-type galaxies, we also started from the G99 compilation (59 galaxies retained), obtained from the $H$ band Tully-Fisher method, with a claimed accuracy of about $16 \%$. We complemented this list with galaxies with $B$-band Tully-Fisher distances from E00 (41) and F98 (109). We do not use galaxies from these lists outside of 8 degrees from M 87 or with morphological types outside of our adopted range (1-9).
To convert E00 distances into the G99 system, two corrections are done: first, we correct the distances to our adopted morphological type, in the 20 cases where it differs from the LEDA type adopted in E00. Indeed, the intercept $b$ of the $B$-band Tully-Fisher relation derived by Theureau et al. (1997) and adopted in E00 depends upon the morphological type. The correction is given by:

$d(T)=d_{\mathrm{E} 00} \times 10^{0.2 \times\left(b\left(T_{\mathrm{LEDA}}\right)-b(T)\right)}$.

Although the morphological type differences never exceed one unit, the correction can amount to $25 \%$ in distance.

Additionally, E00 distances are based on the Theureau et al. (1997) calibration, which leads to $H_{0} \approx$ $55 \mathrm{~km} \mathrm{~s}^{-1} \mathrm{Mpc}$; as E00 adopt a cosmic velocity of Virgo of $1200 \mathrm{~km} \mathrm{~s}^{-1}$, this implies a Virgo distance of $21.8 \mathrm{Mpc}$. Therefore, the conversion factor to the G99 assumed Virgo distance of $16 \mathrm{Mpc}$ is:

$d_{\mathrm{E} 00}^{\mathrm{cor}}=0.733 d_{\mathrm{E} 00}$.

To convert F98 distances into the G99 system, we simply apply the calibration correction derived from different adopted Virgo distances, namely 21.5 Mpc and $16 \mathrm{Mpc}$ :

$d_{\mathrm{F} 98}^{\mathrm{cor}}=0.744 d_{\mathrm{F} 98}$.

As we have 65 galaxies with more than one distance estimate, we can make an intercomparison among the three sources of distance data, after their reduction to the mean system. We find:

$$
\begin{aligned}
& \left\langle d_{\mathrm{E} 00}^{\mathrm{cor}}-d_{\text {mean }}\right\rangle=1.21 \pm 0.29 \\
& \left\langle d_{\mathrm{F} 98}^{\mathrm{cor}}-d_{\text {mean }}\right\rangle=-1.89 \pm 0.18 \\
& \left\langle d_{\mathrm{G} 99}-d_{\text {mean }}\right\rangle=1.13 \pm 0.25
\end{aligned}
$$

where $d_{\text {mean }}$ is the mean value of available distance measurements for a given galaxy.

All these shifts are significant. This means that our first guess of the conversion factors to the G99 system was not as successful as we could hope. We therefore repeated it with new conversion factors, until we reach on average negligible shifts of the corrected systems to the mean one. This leads to the following adopted conversion factors, after rejection of two galaxies with discrepant distance measurements (NGC 4180 and NGC 4591):

$$
\begin{aligned}
& d_{\mathrm{E} 00}^{\text {cor }}=0.678 d_{\mathrm{E} 00} \\
& d_{\mathrm{F} 98}^{\text {cor }}=0.832 d_{\mathrm{F} 98} \\
& d_{\mathrm{G} 99}^{\text {cor }}=0.930 d_{\mathrm{G} 99} .
\end{aligned}
$$

We adopt these new conversion factors to put all three references into the same system. Then, considering that the advantages of the $H$-band (low extinction correction) are compensated by the smaller slope of the Tully-Fisher relation in $B$-band, we do not weigh each measurement and adopt a straight average of the available distance estimates for each galaxy. We will only use these mean distances to classify the galaxies among the various classes described in 

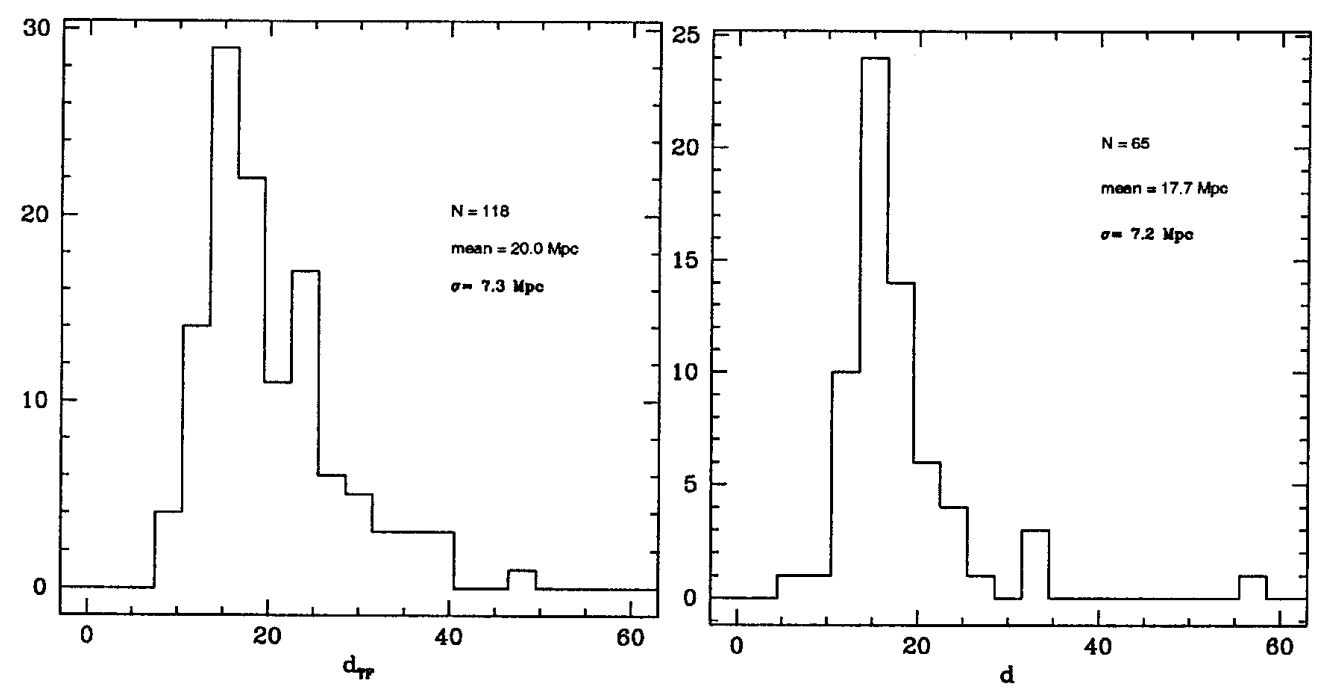

Fig. 2. Histogram of the adopted distances for the 118 spiral galaxies (left) and the 65 early-type galaxies (right).

Sect. 6. We display in Fig. 2 a histogram of these 118 average distances. The mean value of $20 \mathrm{Mpc}$ and the skewness of the histogram clearly show the background contamination of our sample. By comparison, the histogram of distances for early-type galaxies appears to be less contaminated by background galaxies (smaller mean distance and smaller skewness).

\subsection{HI deficiency}

As we are concerned with the distance determination of individual galaxies in the Virgo cluster, we quantify HI deficiency by means of a distance-independent parameter DEF, based on the difference between the expected and observed logarithm of the mean (hybrid) HI surface density, $\Sigma_{\mathrm{HI}}$, that is

$\mathrm{DEF}=\left\langle\log \Sigma_{\mathrm{HI}}\left(T^{\mathrm{obs}}\right)\right\rangle-\log \Sigma_{\mathrm{HI}}^{\mathrm{obs}}$,

with $\Sigma_{\mathrm{HI}}=F_{\mathrm{HI}} / a_{\mathrm{opt}}^{2}$, and where $F_{\mathrm{HI}}$ represents the corrected $\mathrm{HI}$ flux density integrated over the profile width in units of $\mathrm{Jy} \mathrm{km} \mathrm{s}^{-1}$ and $a_{\text {opt }}$ represents the apparent optical diameter in arcmin. For non-detections, an upper limit to HI content is estimated by assuming that the emission profile is rectangular with an amplitude 1.5 times the rms noise and width equal to that expected for a galaxy of the same morphological type and luminosity, properly corrected for redshift broadening and viewing inclination (Solanes et al. 1996; see also Haynes \& Giovanelli 1984). The adopted standard values of $\left\langle\log \Sigma_{\mathrm{HI}}\right\rangle$ per type are: 0.24 for Sa, Sab; 0.38 for Sb; 0.40 for Sbc; 0.34 for Sc; and 0.42 for later spiral types.

Among our sample of 118 spiral galaxies, we have HI deficiency estimates for 106 galaxies, or $90 \%$ of the sample.

It has been suggested that Tully-Fisher distances may be underestimated for highly HI deficient galaxies (T92; Fukugita et al. 1993). For this reason, we have investigated possible differences in the rotation velocities of $\mathrm{HI}$ deficient and $\mathrm{HI}$ normal galaxies in the $12 \mathrm{HI}$ deficient clusters identified by Solanes et al. (2001), as well as the influence of the HI content on the Tully-Fisher relationship of the galaxies in our sample. The results obtained allow us to conclude that HI deficiency does not affect our Tully-Fisher distances.

\section{Tolman-Bondi model}

We use the Tolman-Bondi model of the Virgo cluster as defined in E99. Let us recall that the Tolman-Bondi model gives an analytical solution to Einstein's field equations for a spherically symmetric pressure-free density excess, embedded in an otherwise homogeneous universe. The parameters of the model are: the observed Virgo cluster velocity in the Local Group reference frame $\left(980 \mathrm{~km} \mathrm{~s}^{-1}\right)$, the Virgocentric infall velocity of the Local Group (220 $\mathrm{km} \mathrm{s}^{-1}$ ), the Virgocentric density profile slope $(\alpha=2.85)$, and the deceleration parameter of the background homogeneous universe, taken as an Einstein de Sitter universe for simplicity $\left(q_{0}=0.5\right)$. Although there is a general agreement about the values of 3 of these parameters, the Virgocentric density profile slope is not well known, as it should reflect the distribution of the mass around the cluster center, not only the galaxy (light) distribution. In E99, it has been constrained using 32 galaxies whose distances were known using their Cepheids, generally measured with the HST, through the PL-relation. For details about the model, the adjustment of its parameters and the influence of changing these values, the reader is referred to T92, E99 and E00.

For each galaxy, the angular distance to the Virgo center of mass (taken to be the position of M 87) completely defines the exact shape of the Tolman-Bondi S-curve in the velocity distance diagram, for a given choice of model parameters. Then, the observed recession velocity of the galaxy (in the Local Group reference frame) gives one to three possible distance ratios of the galaxy to Virgo (see Fig. 3 for examples). The first and the third values correspond to a galaxy falling into the cluster from in front 

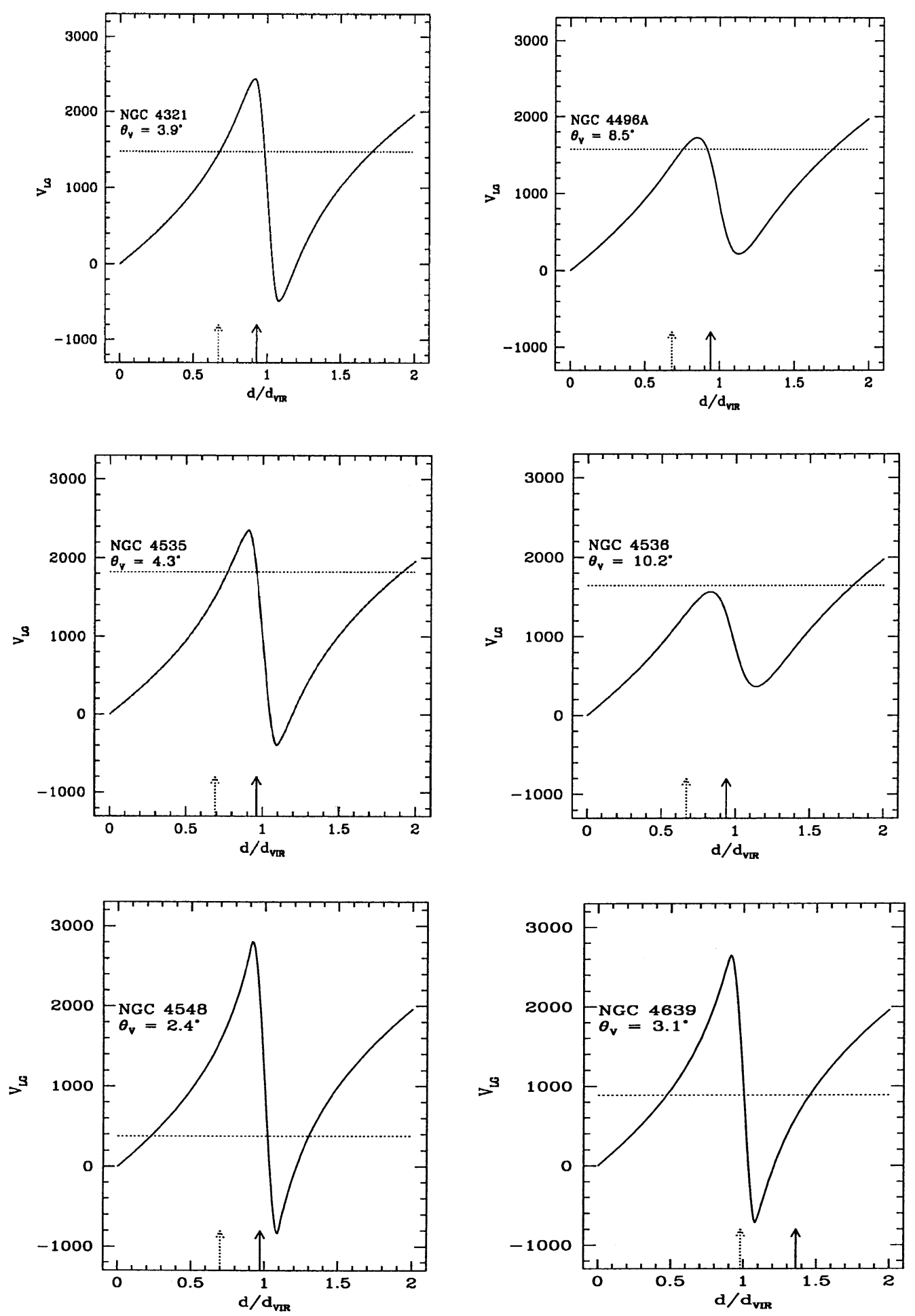

Fig. 3. Tolman-Bondi model for the six galaxies with Cepheid distances. The dotted line corresponds to the recession velocity of the galaxy, while the arrows mark the expected distance ratio if $R_{\mathrm{VIR}}=15.4 \mathrm{Mpc}$ ( solid arrow) or $R_{\mathrm{VIR}}=21.5 \mathrm{Mpc}(\mathrm{dotted}$ arrow).

or behind. The second value corresponds to a true cluster member. Assuming that the Tolman-Bondi model can explain most of the observed positions of the galaxies in the velocity-distance diagram means that we neglect other possible components, such as an expanding one suggested by $\mathrm{T} 92$, or galaxies projected by chance onto the cluster, but taking part in the Hubble flow. Certainly, not all galaxies in the Virgo cluster should follow the TolmanBondi model. For instance, galaxies belonging to the virialized core of the cluster do not exhibit a velocity-distance relation. It is however difficult to disentangle those galaxies from our class 2 , so that we include them into it. On the 
other hand, not all galaxies in the class 2 (defined as the descending branch of the S-shape Tolman-Bondi curve) belong to Virgo: for instance, at large angular distances from M 87 the descending branch still exists, but is no longer related to proper Virgo galaxies. We assume that within 8 degrees of the cluster center, most of the galaxies on the descending branch are true cluster members. We will test this strong assumption in Sect. 6 by building a histogram of "Virgo distances", where each point is computed from a galaxy distance and its distance ratio to Virgo, as given by the Tolman-Bondi model.

To do this, we must select the most plausible one among the possible distance ratios for a given galaxy, when the model gives more than one value. Here, we made use of two criteria: we try to choose the value closest to the assumed Virgo distance (16 Mpc), and, for spiral galaxies, we take into account the HI deficiency, assuming that a true member of the cluster has a higher probability to be deficient than a galaxy falling for the first time into the cluster. We therefore attribute to each galaxy a "class number", which is 1 if we adopt the first value of the distance ratio to Virgo (galaxies falling from in front into the cluster), 2 if we adopt the second value (true cluster members), and 3 if we adopt the third value (galaxies falling from behind into the cluster).

\section{Distance to the Virgo cluster from Cepheids}

There are 6 galaxies in or close to the Virgo cluster whose distance is known thanks to the HST observations in $V$ and $I$ bands, using the Cepheid period-luminosity relation. We use the distances published in Freedman et al. (2001) (without the uncertain metallicity correction). Five of the six distances cluster about a mean distance of 14.6 Mpc, while the sixth one (NGC 4639) gives a larger distance $(21 \mathrm{Mpc})$. This is perfectly explained by the Tolman-Bondi model, and this was in fact the initial motivation of this study. Figure 3 gives the result of the application of the Tolman-Bondi model to these galaxies, with a dotted line showing the recession velocity of the galaxy, and an arrow showing the distance ratio to Virgo, for an adopted distance to Virgo of $15.4 \mathrm{Mpc}$ (see below).

Two galaxies belong to the Virgo southern extension (NGC 4496A and NGC 4536) and lie at more than 8 degrees from M 87 . However, their position in the velocitydistance diagram should be explained by our model, if we assume that no other cluster perturbes them. However, we find that the maximum velocity explained by our model for NGC 4536 is $1563 \mathrm{~km} \mathrm{~s}^{-1}$ at $0.83 d_{\text {Virgo }}$, too small compared to its observed velocity of $1641 \mathrm{~km} \mathrm{~s}^{-1}$. For this galaxy, our model predicts it to be in the class 3 , with a distance of $1.79 d_{\text {Virgo }}$. This in turn would lead to an inacceptable Virgo distance of $d_{\mathrm{Virgo}}=8.07 \mathrm{Mpc}$, using the observed galaxy distance of $14.45 \mathrm{Mpc}$. In fact, it is well known that random velocities about $80 \mathrm{~km} \mathrm{~s}^{-1}$ exist for all galaxies and explain the velocity dispersion of small groups of galaxies (Gourgoulhon et al. 1992). We therefore assume that this is the origin of the small discrepancy observed for NGC 4536.

Table 1 gives the adopted distances to the six galaxies, the model predicted distance ratios to the Virgo distance for the three possible solutions, the corresponding predicted "Virgo distances", the adopted class, and the adopted Virgo distance for each galaxy. Now we discuss each galaxy in some detail: for NGC 4548, only the second and third distances are plausible; but the large HI deficiency leads us to attribute this galaxy to class 2 as a true member of the Virgo cluster. Similarly, the large HI deficiency of NGC 4321 makes us attribute it to class 2. On the contrary, the insignificant deficiency of NGC 4639 leads to put it into class 3 , with a derived Virgo distance intermediate between NGC 4321 and NGC 4548. For NGC 4535, the HI deficiency is low, but putting it into class 1 would lead to a larger derived Virgo distance than the previous galaxies, so we attribute it to class 2. NGC 4496A belongs to the Virgo southern extension and is not HI deficient; it is therefore tempting to put it into class 1 , but again putting it into class 2 leads to a derived Virgo distance in better agreement with the remaining galaxies. Finally, NGC 4536 also belongs to the Virgo southern extension and has a small HI deficiency, and it may be put into class 1 or 2, once its recession velocity is corrected for a random component. The resulting mean value of the six derived Virgo distances is $15.4 \pm 0.5 \mathrm{Mpc}$, which we adopt as our estimate of the cluster distance.

At the referee's request, we have investigated what happens if a Virgo distance of $21.5 \mathrm{Mpc}$ is preferred from independent arguments. Then, four of the five galaxies classified in class 2 now fall into class 1 (infalling galaxies), NGC 4639 is the only true member of the Virgo cluster among the six, and NGC 4548 position cannot be explained by the model. The high HI deficiency of NGC 4321 is contradictory. This alternative distance is clearly less probable according to these six galaxies with Cepheid distances. A definitive answer will only become available with a larger sample of Virgo galaxies with accurate distances.

\section{Mass of the Virgo cluster}

Derivation of the Virgo cluster mass from the TolmanBondi model, and its comparison with the virial mass estimate, have been discussed in T92, E99 and E00. Here, we give a summary of the useful formulae and derive the Virgo mass for our adopted model parameters. Let $R_{\mathrm{VIR}}$ be the Virgo distance in Mpc, $V_{\mathrm{VIR}}^{\mathrm{cosm}}$ the cosmic recession velocity of Virgo in $\mathrm{km} \mathrm{s}^{-1}$ and $d$ the radius normalized to the Virgo distance. The mass enclosed within $d$ is the product of the "Einstein - de Sitter mass" within the same radius by the mass excess due to the cluster. It is given in solar mass units by:

$M(d)=M(d)_{\mathrm{EdS}} \times\left(1+k^{\prime} d^{-\alpha}\right)$

$M(d)_{\mathrm{EdS}}=2.32510^{8} q_{0} R_{\mathrm{VIR}}\left(V_{\mathrm{VIR}}^{\mathrm{cosm}}\right)^{2} d^{3}$. 
Table 1. Adopted Cepheid distances to the 6 Virgo galaxies, ratio to the Virgo distance given by the Tolman-Bondi model and corresponding Virgo distances for each possible class, adopted class and Virgo distance, and HI deficiency.

\begin{tabular}{|c|c|c|c|c|c|c|c|}
\hline \multirow[t]{2}{*}{ Name } & \multirow{2}{*}{$\begin{array}{c}d \\
\mathrm{Mpc}\end{array}$} & \multicolumn{3}{|c|}{$d / d_{\mathrm{V}}$} & \multirow[t]{2}{*}{ class } & \multirow{2}{*}{$\begin{array}{c}d_{\mathrm{V}} \\
\mathrm{Mpc}\end{array}$} & \multirow[t]{2}{*}{ def } \\
\hline & & $d_{1}$ & $d_{2}$ & $d_{3}$ & & & \\
\hline \multirow[t]{2}{*}{ NGC 4321} & 14.32 & 0.68 & 0.95 & 1.71 & 2 & 15.07 & 0.49 \\
\hline & & 21.06 & 15.07 & 8.37 & & & \\
\hline \multirow[t]{2}{*}{ NGC 4496A } & 14.52 & 0.75 & 0.92 & 1.76 & $2(1)$ & 15.78 & -0.09 \\
\hline & & 19.36 & 15.78 & 8.25 & & & \\
\hline \multirow[t]{2}{*}{ NGC 4535} & 14.79 & 0.77 & 0.96 & 1.91 & $2(1)$ & 15.41 & 0.19 \\
\hline & & 19.21 & 15.41 & 7.74 & & & \\
\hline \multirow[t]{2}{*}{ NGC 4536} & 14.45 & $(0.83)$ & $(0.83)$ & 1.79 & 1 or 2 & $(17.41)$ & 0.25 \\
\hline & & $(17.41)$ & (17.41) & 8.07 & & & \\
\hline \multirow[t]{2}{*}{ NGC 4548} & 15.00 & 0.23 & 1.07 & 1.30 & 2 & 14.02 & 0.83 \\
\hline & & 65.22 & 14.02 & 11.54 & & & \\
\hline \multirow[t]{2}{*}{ NGC 4639} & 20.99 & 0.47 & 1.03 & 1.45 & 3 & 14.48 & 0.10 \\
\hline & & 44.66 & 20.38 & 14.48 & & & \\
\hline
\end{tabular}

$k^{\prime}$, the mass excess within $d=1$, i.e. at the Local Group location, only depends on the observed Virgo cluster velocity, the Virgocentric infall velocity of the Local Group and the cosmological parameters of the adopted background universe $\left(q_{0}\right.$ and $\left.H_{0} \times t_{0}\right)$. It does not depend on the Virgocentric density profile slope $\alpha$. Its value is the same as in E99, namely $k^{\prime}=0.606$. For Virgo, we define the radius $d$ as corresponding to 8 degrees, which gives $d=0.141$, in place of 0.105 , corresponding to 6 degrees in E99.

With $V_{\mathrm{VIR}}^{\text {cosm }}=1200 \mathrm{~km} \mathrm{~s}^{-1}$ and $R_{\mathrm{VIR}}=15.4 \mathrm{Mpc}$, we get $M=1.2 \times 10^{15} M_{\odot}$. The virial mass of Virgo as given by Tully \& Shaya (1984) is:

$M_{\text {virial }}=2.325 \times 10^{8} \times\left(\pi R_{\Omega}\right) \times\left(3 \sigma_{\mathrm{V}}^{2}\right)$

where notations are those from Tully \& Shaya. Their result transposed to $15.4 \mathrm{Mpc}$ gives $M_{\text {virial }}=6.9 \times 10^{14} M_{\odot}$, so that $M=1.7 M_{\text {virial }}$. E99 found a coefficient 1.62 for their adopted parameters of the Tolman-Bondi model.

By comparison, Böhringer et al. (1994) estimate the mass of the M 87 sub-cluster from X-ray emission measured by ROSAT to $\sim(1.5-6) \times 10^{14} M_{\odot}$ within a radius of $1.8 \mathrm{Mpc}$ at $20 \mathrm{Mpc}$ (5 degrees). Contributions from the M 49 and M 86 sub-clusters are negligible, at about $(1-3) \times 10^{13} M_{\odot}$. These values are confirmed by Schindler et al. (1999) who derive $2.1 \times 10^{14} M_{\odot}$ within $1.5 \mathrm{Mpc}$ around M 87 (at $20 \mathrm{Mpc}$ ) and $0.87 \times 10^{14} M_{\odot}$ within $0.75 \mathrm{Mpc}$ around M 49. Our Tolman-Bondi mass for the same distance would be $M=1.5 \times 10^{15} M_{\odot}$, almost one order of magnitude larger. We do not have any explanation to offer for this discrepancy, but we note that our large mass estimate and steep density profile are supported by Tully \& Shaya (1998), who find a Virgo mass of $M=1.3 \times 10^{15} M_{\odot}$ from a modeling of the velocity field of the Local Supercluster, assuming a mass-to-light ratio of $150 M_{\odot} / L_{\odot}$ in the field, but 1000 for the Virgo cluster, and $\Omega_{0}=0.3^{1}$.

\section{Structure of the Virgo cluster}

Disentangling the different components of the Virgo cluster is difficult and somewhat subjective. However, the use of the Tolman-Bondi model allows us to classify each galaxy into one of the three classes defined previously. We did that independently for early-type and spiral galaxies. In each case, we have built several diagrams: the first one is the histogram of the "Virgo derived distances", and it is shown in Fig. 4. Both histograms now have a well-defined peak around the assumed Virgo distance and are symmetric, a posteriori confirming our hypothesis. Mean distances in both cases are compatible with the assumed distance of $16 \mathrm{Mpc}$. However, the rms dispersion is slightly lower in the case of early-type galaxies: this may reveal that the spiral sample class attribution is still not fully satisfying, or that our restrictive hypothesis rejecting expanding components or projected galaxies in the Tolman-Bondi model are not completely fulfilled.

The second diagram gives the velocity repartition among the different classes, and is displayed in Fig. 5. In fact, we have split class 3 into two classes, because it clearly contains high velocity objects (class 4 , above $1600 \mathrm{~km} \mathrm{~s}^{-1}$ ), which are background galaxies, and low velocity objects (class $3,0-1300 \mathrm{~km} \mathrm{~s}^{-1}$ ), which are infalling galaxies from behind the cluster. We have not convincingly identified any spiral galaxy belonging to class 1 ; the only two early-type objects classified in class 1 exhibit low velocities (about $800 \mathrm{~km} \mathrm{~s}^{-1}$ ), which is difficult to interpret; in fact, these two galaxies may be genuine members of the cluster (class 2) with bad distance measurements;

\footnotetext{
1 Although Tully \& Shaya do not specify their adopted distance to Virgo, we assume it is the same as in Tully \& Shaya (1984), namely $16.8 \mathrm{Mpc}$.
} 


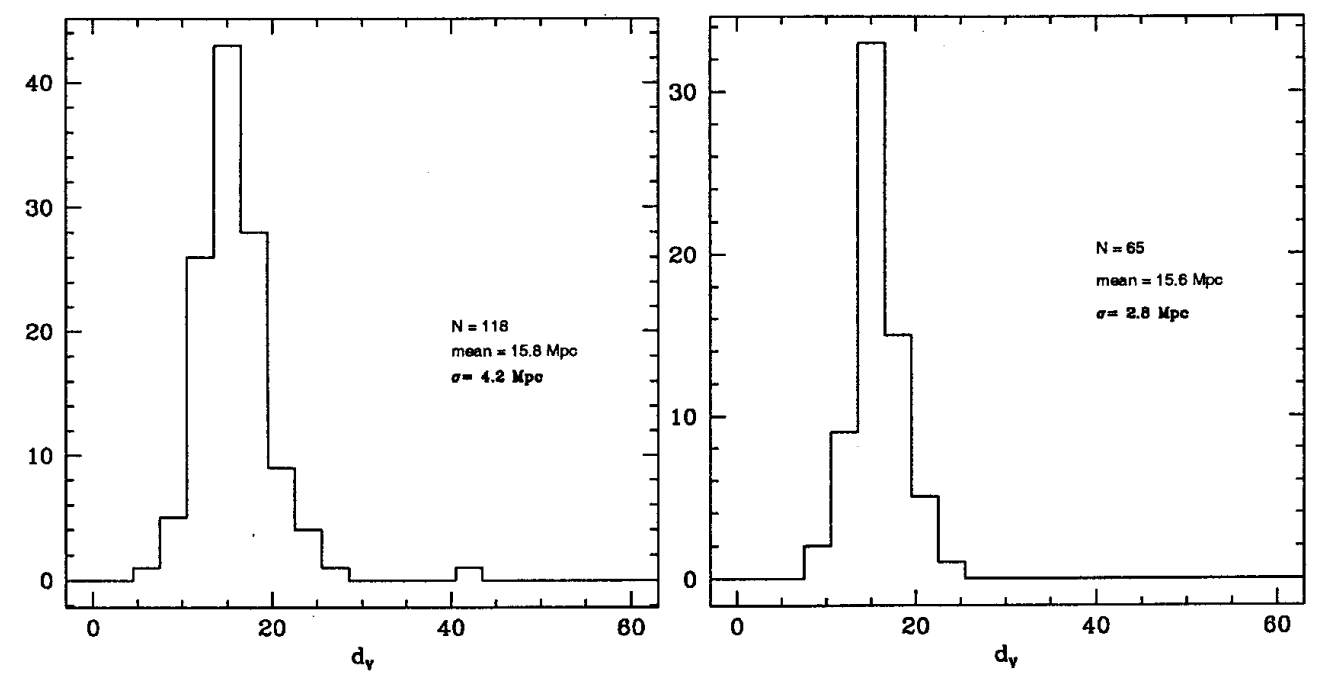

Fig. 4. Histogram of the "Virgo derived distances" for spiral galaxies (left) and early-type galaxies (right).
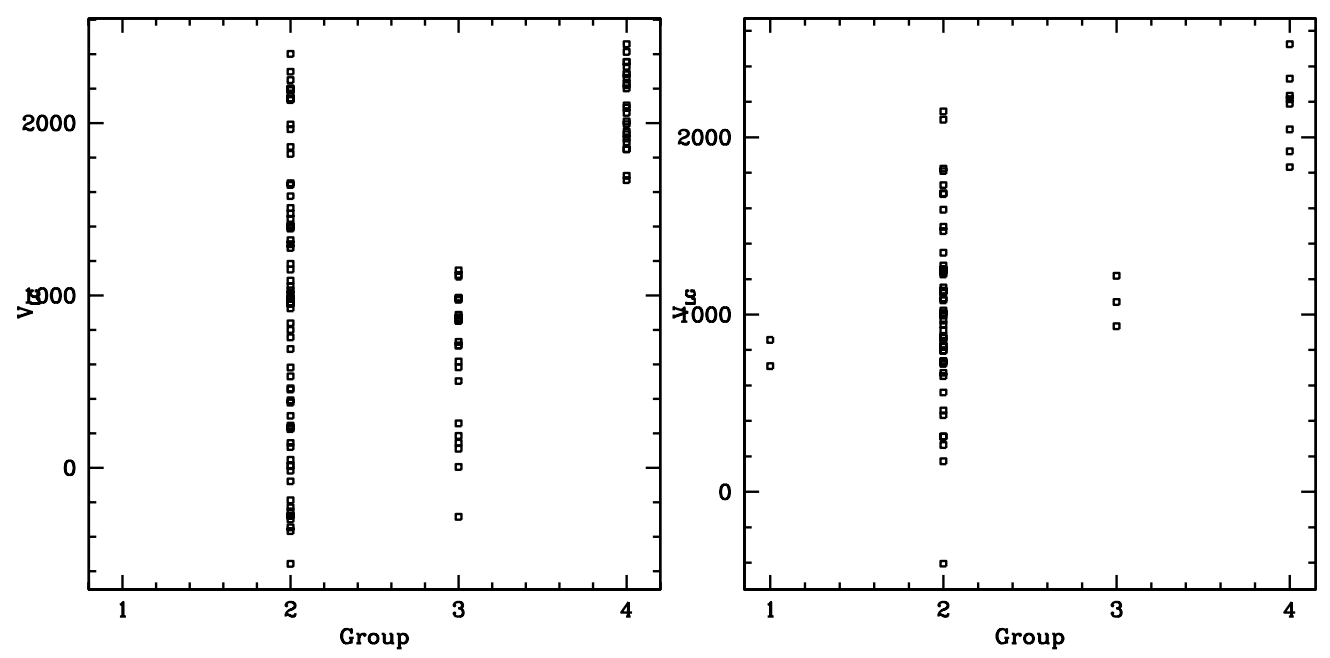

Fig. 5. Distribution of the recession velocities among the different classes for spiral galaxies (left) and early-type galaxies (right).

indeed, both galaxies have a velocity dispersion smaller than $100 \mathrm{kms}^{-1}$, which makes their fundamental plane distances quite uncertain.

A third diagram gives the repartition of $\mathrm{HI}$ deficiency among the classes, obviously only for spiral galaxies. It is displayed in Fig. 6. It is clear that there is no highly deficient galaxies among classes 1, 3 and 4, as expected because we used HI deficiency as one of our classification criteria.

Finally, Fig. 7 displays the repartition on the sky of spirals and early-type galaxies with different symbols for different classes. If we compare the distribution of the two types of galaxies, it clearly appears that the different classes concentrate into common regions. One can easily recognize the $\mathrm{W}$ group around $\left(12.3 \mathrm{~h}, 6^{\circ}\right)$ and the $\mathrm{M}$ group around $\left(12.2 \mathrm{~h}, 13^{\circ}\right)$. Both groups have a mean distance ratio to Virgo of 2.1. Galaxies at the same distance but slightly displaced from the main concentration have been classified as group halo. Finally, galaxies with intermediate distances between those of these groups and the Virgo distance, and classified into our class 3, are

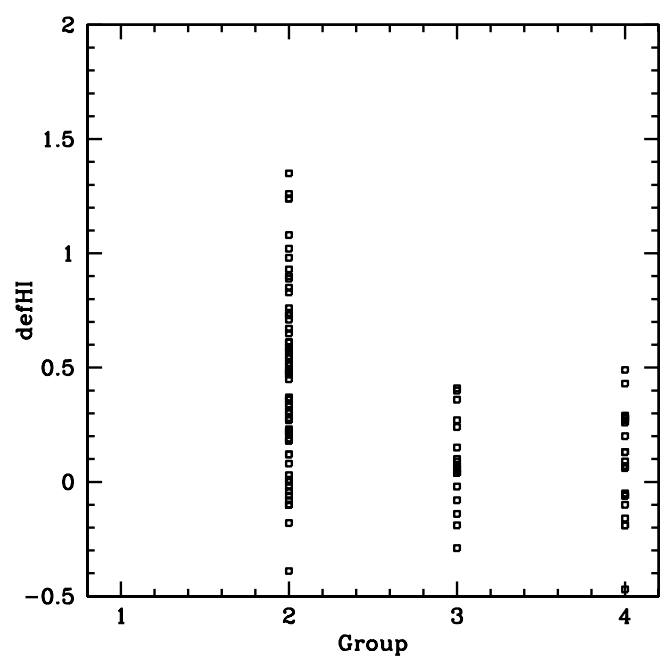

Fig. 6. Distribution of HI deficiency among the different classes for spiral galaxies.

interpreted as belonging to extended filaments extracted from the background group and falling into Virgo. Such 

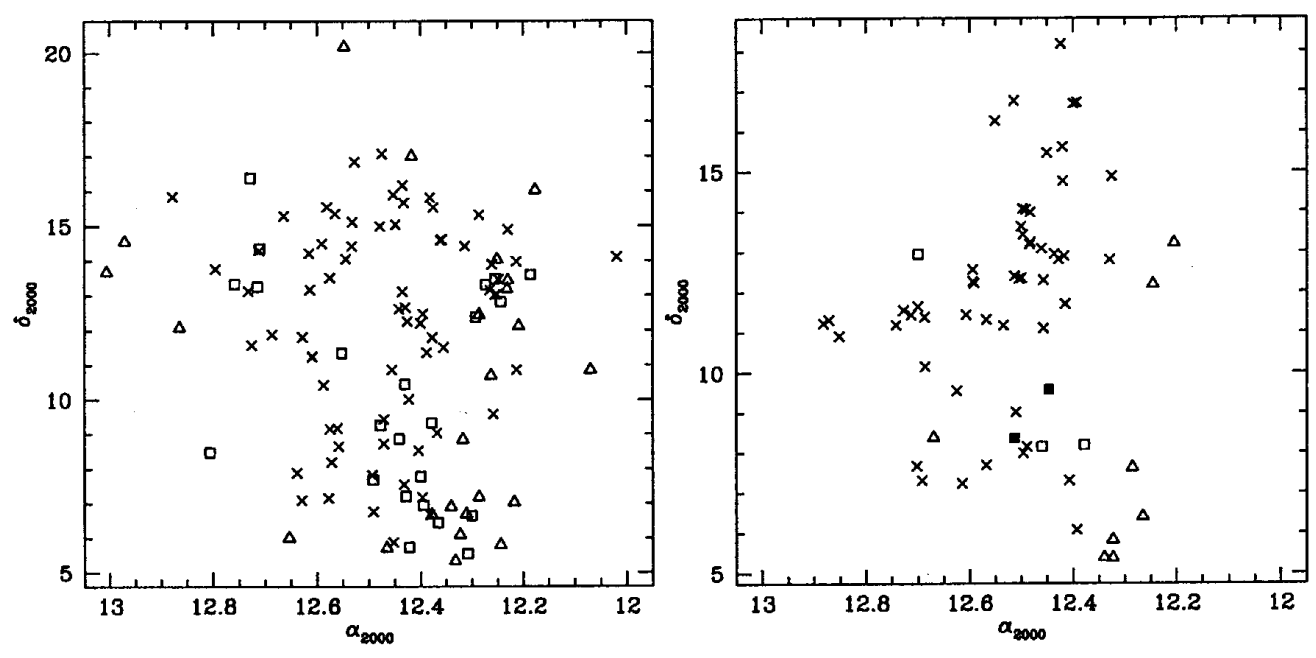

Fig. 7. Distribution on the sky of the spiral galaxies (left) and early-type galaxies (right). Filled squares correspond to class 1 , crosses to class 2 , open squares to class 3 and open triangles to class 4 .

is the case of the NGC 4222 group, which may link the $\mathrm{M}$ group to Virgo at a mean distance ratio to Virgo of 1.2, and the NGC 4343 group, around (12.4 h, $8^{\circ}$ ), similar to the B group of G99 and introduced by de Vaucouleurs (1961) as its W' group, and which may link the W group to the Virgo cluster at a mean distance ratio to Virgo of 1.5. A small group around NGC 4639 at $\left(12.7 \mathrm{~h}, 14^{\circ}\right)$ can also be identified with the eastern group of G99, although our group is less extended and less numerous; it could be linked to a remote group of galaxies around NGC 4746 .

In Table 2, we propose a new nomenclature for all these groups, based on the name of its main galaxy, together with a list of the members we identified in this study. These lists are obviously not complete, because of the lack of distance measurements of some other well-known members. Table 3 lists other galaxies which have not been attributed to these groups nor to the main cluster, and are classified as background (class 4), infalling from behind (class 3), or from in front (class 1).

After excluding all the galaxies which do not belong to the main cluster according to our classification, we are left with 71 spiral and 52 early-type galaxies. $67 \%$ of the original sample with distances are therefore considered as true members of the cluster; not surprisingly, the spiral sample exhibits a larger contamination $(40 \%)$ than the early-type sample (20\%). Among our 5 references, the proportion of true members varies from $56 \%$ (E00) and 61\% (F98) to $83 \%$ (T01).

\section{Comparison of Virgo distances}

Now that we have classified all the galaxies of our sample into the different classes, we can return to the different distances adopted by the various authors to the Virgo cluster.

We will first derive an average ratio of distances from a given reference to the predicted distance given by the Tolman-Bondi model and an adopted Virgo distance of
15.4 Mpc. For the $i$ th galaxy measured by reference $j$, we have:

$$
\begin{aligned}
r_{i j} & =\frac{d_{i j}}{\left(d / d_{\mathrm{V}}\right)_{i} \times 15.4} \\
r_{j} & =\left\langle r_{i j}\right\rangle .
\end{aligned}
$$

In the second test we restrict our sample to true Virgo galaxies (class 2) and compute the mean distance for each reference. The results will depend much less on our adopted Tolman-Bondi model but should confirm it.

Table 4 gives the results. We can see that for G99 and T01, the agreement between the resulting mean Virgo distances from both methods (Cols. 3 and 4, respectively) and the value adopted by these authors (Col. 5) is satisfying. On the contrary, for F98 the resulting mean Virgo distances are consistent from both methods, but smaller than their adopted value: this is certainly due to inclusion of background galaxies into their sample; indeed, among the 49 galaxies of their "fiducial sample", 14 are classified by us as non-members of the Virgo cluster. For E00 the result is inverse and the mean error is large: it seems therefore that there is a discrepancy between their adopted Hubble constant (from Theureau et al. 1997) and the Virgo distance we derive from their data.

For these two references, we adopt as the mean Virgo distance the average over the two determinations (Cols. 3 and 4). This gives 18.9 and $23.2 \mathrm{Mpc}$ for F98 and E00, respectively. If we apply to these distances the conversion factors adopted in Sect. 2.3.2 to reduce F98 and E00 to a mean Virgo distance of $16 \mathrm{Mpc}$, namely 0.832 and 0.678 respectively, we now get values close to $16 \mathrm{Mpc}$ (15.7 and 15.8 Mpc, respectively).

However, it is important to keep in mind that the clean samples of true members may still be affected by the incompleteness bias (Fouqué et al. 1990 for the specific case of the Virgo cluster; Teerikorpi 1997 for a general discussion), which may lead to average cluster distances which are too short. To estimate the amount of this bias, we 
Table 2. List of groups outside of the main Virgo cluster, with their mean distance ratio to Virgo and a list of members with known distance.

\begin{tabular}{lcll}
\hline Name & $d / d_{\mathrm{v}}$ & Location & Members \\
\hline NGC 4168 & 2.13 & M group & NGC 4168, NGC 4189, NGC 4193, NGC 4200, IC 769, IC 3061, IC 3099 \\
& 2.07 & M halo & NGC 4067, NGC 4152, IC 3074 \\
NGC 4222 & 1.24 & M infall & NGC 4222, IC 3033, IC 3066, IC 3105, UGC 7249 \\
NGC 4261 & 2.11 & W group & NGC 4180, NGC 4197, NGC 4215, NGC 4233, NGC 4235, NGC 4259, NGC 4260, \\
& & & NGC 4261, NGC 4273, NGC 4281, IC 3225, CGCG 42-1, CGCG 42-36 \\
& 2.17 & W halo & IC 776, UGC 7579 \\
NGC 4343 & 1.46 & W infall & NGC 4252, NGC 4316, NGC 4318, NGC 4343, NGC 4353, NGC 4376, NGC 4390, \\
& & & NGC 4411A, NGC 4434, NGC 4451, NGC 4466, IC 3115, IC 3322A, UGC 7423 \\
NGC 4639 & 1.41 & & NGC 4620, NGC 4633, NGC 4639, NGC 4651, IC 3742 \\
NGC 4746 & 1.92 & & NGC 4746, UGC 8085, UGC 8114 \\
\hline
\end{tabular}

Table 3. List of galaxies outside the main Virgo cluster and not attributed to any group, with their distance ratio to Virgo.

\begin{tabular}{lcc}
\hline Name & $d / d_{\mathrm{V}}$ & Class \\
\hline NGC 4417 & 0.39 & 1 \\
NGC 4488 & 0.46 & 1 \\
NGC 4591 & 2.21 & 4 \\
NGC 4598 & 1.92 & 4 \\
NGC 4698 & 1.44 & 3 \\
IC 3298 & 2.27 & 4 \\
IC 3483 & 1.22 & 3 \\
UGC 7697 & 2.34 & 4 \\
\hline
\end{tabular}

have calibrated once more the $B$-band Tully-Fisher relation using the 21 calibrators from Freedman et al. (2001), and the 51 true members of Virgo according to our classification, which best match the calibrator properties (thus excluding peculiar, interacting, HI-truncated galaxies, and restricting to morphological types between 2 and 8, inclinations between 37 and 90 degrees, $\log V_{\max }$ larger than 1.7), with data extracted from the LEDA database. As usual, the slope of the Tully-Fisher relation is determined from the Virgo sample (more numerous), and the intercept from the calibrators. The rms dispersion is similar for both samples ( 0.41 for calibrators, 0.53 for Virgo sample). The direct fit (appropriate to distance determinations) gives:

$M_{B}=-6.28( \pm 0.39) \log V_{\max }-6.31( \pm 0.09)$.

The intercept for the Virgo sample, $24.96 \pm 0.11$ gives a distance modulus of $31.27 \pm 0.14$, or a distance of $18.0 \pm 1.2 \mathrm{Mpc}$, which is larger than our adopted distance from Cepheids. If we divide the sample of 51 Virgo galaxies equally into large and small $\log V_{\max }$, we get mean distances of:

$\mu=31.24 \pm 0.09$, for $\log V_{\max }>1.992$

$\mu=31.28 \pm 0.12$, for $\log V_{\max } \leq 1.992$.

It can therefore safely be concluded that the incompleteness bias does not affect significantly our sample of true Virgo members (it would lead to a smaller mean distance for galaxies with small rotation velocities, which are more biased).

It can still be argued that the bias may be hidden, because the slope of the Tully-Fisher relation, when determined from the Virgo cluster sample, may be biased due to a possible incompleteness of the sample at small $\log V_{\max }$. This is however not observed, as the direct slope determined from the calibrators is $-5.24 \pm 0.66$, while an incompleteness bias would predict a steeper slope as compared to the slope determined from the Virgo sample $(-6.28 \pm 0.39)$.

The 0.3 mag difference between the Cepheid and TullyFisher distances is worrying. Tenants of the long distance scale will see evidence that the Tully-Fisher distance is the correct one, while most galaxies with Cepheid distances are infalling on the front side of the cluster.

\section{Conclusions}

In this paper, we have investigated how the relativistic Tolman-Bondi model as applied in E99 gives constraints on the Virgo cluster mass and distance, and allows one to disentangle its quite intricate structure. Distances to 183 Virgo galaxies from 5 references have been used and averaged, together with HI deficiency parameters for spirals, to classify the galaxies into 4 different distance classes: an infalling component in front of Virgo, the Virgo cluster itself, an infalling component behind the main cluster, and background groups. The main results of the present study are:

- Among the 6 galaxies in the Virgo area with Cepheid distance measures, NGC 4639 is well-known to be discrepant. Our model nicely explains this apparent discrepancy, and the resulting mean distance to Virgo using all 6 calibrators is $15.4 \pm 0.5 \mathrm{Mpc}$. However, the mean Tully-Fisher distance derived from 51 spiral galaxies classified as true members of the cluster in the present study, and calibrated using the same Cepheid distance system (Freedman et al. 2001) is larger $(18.0 \pm 1.2 \mathrm{Mpc})$;

- The mass of the Virgo cluster derived from our model is large: $M=1.2 \times 10^{15} M_{\odot}$, which corresponds to 
Table 4. Mean ratio of galaxy distances to Tolman-Bondi predictions for $d_{\text {Virgo }}=15.4$ Mpc, resulting mean Virgo distance, comparison with the mean distance restricted to true Virgo galaxies and to the distance adopted by each of our 5 references.

\begin{tabular}{lcccc}
\hline Reference (Galaxy type) & Ratio & $d_{\mathrm{V}}$ & $d_{\mathrm{V}}^{\text {true }}(N, \sigma)$ & $d_{\mathrm{V}}^{\text {ref }}$ \\
\hline G99 (E) & $1.017 \pm 0.031$ & $15.7 \pm 0.5$ & $15.3 \pm 0.5(36,2.94)$ & 16.0 \\
T01 (E) & $1.084 \pm 0.024$ & $16.7 \pm 0.4$ & $17.0 \pm 0.4(29,1.99)$ & $17.0 \pm 0.3$ \\
G99 (S) & $1.025 \pm 0.025$ & $15.8 \pm 0.4$ & $15.7 \pm 0.5(41,3.10)$ & 16.0 \\
F98 (S) & $1.221 \pm 0.027$ & $18.8 \pm 0.4$ & $19.1 \pm 0.5(67,4.27)$ & $21.5 \pm 2.3$ \\
E00 (S) & $1.487 \pm 0.053$ & $22.9 \pm 0.8$ & $23.6 \pm 1.1(23,5.51)$ & 21.8 \\
\hline
\end{tabular}

1.7 virial mass. Our adopted mass profile is steeper than the light distribution (anti-biasing);

- Apart from the well-known background groups which once contaminated Virgo samples, we have identified a number of galaxies at intermediate distances between these groups and the cluster itself, which we interpret as filaments extracted from the background groups and falling into the cluster from behind;

- We have not been able to securely identify galaxies in front of the cluster and falling into it. This supports Sandage \& Tammann (1976) who always argued that apparently foreground galaxies in the Virgo cluster direction were in fact cluster members;

- Distances to the Virgo cluster adopted by F98 and E00 differ from what would be expected using the distances predicted by our model and the mean ratio of published to predicted distances. They also differ from the mean of the published distances restricted to the true Virgo members. We also note that the distances published in E00 are highly dispersed, even for true Virgo members.

Acknowledgements. We have made use of the LEDA database (http://leda.univ-lyon1.fr), supplied by the LEDA team at the CRAL - Observatoire de Lyon (France). We warmly thank all the LEDA team members for their effort. We also thank Riccardo Giovanelli and Martha Haynes for making their Arecibo General Catalog, from which we have extracted the HI data used in this study, available to us. Finally, we wish to thank the referee, Pekka Teerikorpi, for his very constructive comments. T. S. acknowledges support from a fellowship of the Ministerio de Educación, Cultura y Deporte of Spain. C. B. acknowledges ESO for a visiting position in Santiago during which this work was started.

\section{References}

Binggeli, B., Popescu, C. C., \& Tammann, G. A. 1993, A\&AS, 98,275

Binggeli, B., Sandage, A., \& Tammann, G. A. 1985, AJ, 90, 1681 (VCC)

Böhringer, H., Briel, U. G., Schwarz R. A., et al. 1994, Nature, 368,828

de Vaucouleurs, G. 1961, ApJS, 6, 213

de Vaucouleurs, G., \& de Vaucouleurs, A. 1973, A\&A, 28, 109

de Vaucouleurs, G., de Vaucouleurs, A., Corwin H. G., et al. 1991, Third Reference Catalogue of Bright Galaxies (Springer-Verlag, New-York) (RC3)

Ekholm, T., Lanoix, P., Teerikorpi, P., Paturel, G., \& Fouqué, P. 1999, A\&A, 351, 827 (E99)

Ekholm, T., Lanoix, P., Teerikorpi, P., Fouqué, P., \& Paturel, G. 2000, A\&A, 355, 835 (E00)

Federspiel, M., Tammann, G. A., \& Sandage, A. 1998, ApJ, 495, 115 (F98)

Fouqué, P., Bottinelli, L., Gouguenheim, L., \& Paturel, G. 1990, ApJ, 349, 1

Freedman, W. L., Madore, B. F., Gibson, B. K., et al. 2001, ApJ, 553, 47

Ftaclas, C., Fanelli, M. N., \& Struble, M. F. 1984, ApJ, 282, 19

Fukugita, M., Okamura, S., \& Yasuda, N. 1993, ApJ, 412, L13

Gavazzi, G., Boselli, A., Scodeggio, M., Pierini, D., \& Belsole, E. 1999, MNRAS, 304, 595 (G99)

Gourgoulhon, E., Chamaraux, P., \& Fouqué, P. 1992, A\&A, 255,69

Haynes, M. P., \& Giovanelli, R. 1984, AJ, 89, 758

Kraan-Korteweg, R. C., Cameron, L. M., \& Tammann, G. A. 1988, ApJ, 331, 620

Paturel, G. 1979, A\&A, 71, 106

Pierce, M. J., \& Tully, R. B. 1988, ApJ, 330, 579

Sandage, A., \& Tammann, G. A. 1976, ApJ, 207, L1

Schindler, S., Binggeli, B., \& Böhringer, H. 1999, A\&A, 343,420

Solanes, J. M., Giovanelli, R., \& Haynes, M. P. 1996, ApJ, 461, 609

Solanes, J. M., Manrique, A., González-Casado, G., et al. 2001, ApJ, 548, 97

Teerikorpi, P. 1997, ARA\&A, 35, 101

Teerikorpi, P., Bottinelli, L., Gouguenheim, L., \& Paturel, G. 1992, A\&A, 260, 17 (T92)

Theureau, G., Hanski, M., Ekholm T., et al. 1997, A\&A, 322,730

Tonry, J. L., Dressler, A., Blakeslee, J. P., et al. 2001, ApJ, 546, 681 (T01) (tables also available at http://www.ifa.hawaii.edu/jt/)

Tully, R. B. 1982, ApJ, 257, 389

Tully, R. B., \& Fisher, J. R. 1977, A\&A, 54, 661

Tully, R. B., \& Shaya, E. J. 1984, ApJ, 281, 31

Tully, R. B., \& Shaya, E. J. 1998, in Evolution of Large Scale Structure, ed. R. F. Stein, \& A. G. W. Cameron (ESO, Garching), 333

van den Bergh, S., Pierce, M. J., \& Tully, R. B. 1990, ApJ, 359,4

Yahil, A., Tammann, G. A., \& Sandage, A. 1977, ApJ, 217, 903

Yasuda, N., Fukugita, M., \& Okamura, S. 1997, ApJS, 108, 417 